\title{
Review of Livermore-led neutron capture studies using DANCE
}

\author{
W.E. Parker ${ }^{1}$, S.A. Sheets ${ }^{2}$, U. Agvaanluvsan ${ }^{1}$, J.A. Becker ${ }^{1}$, F. Becvar ${ }^{3}$, T.A. Bredeweg ${ }^{4}$, R. Clement ${ }^{4}$, \\ A. Couture ${ }^{4}$, E. Esch $^{4}$, R.C. Haight ${ }^{4}$, M. Jandel ${ }^{4}$, M. Krticka ${ }^{3}$, G.E. Mitchell ${ }^{5}$, R. Macri ${ }^{1}$, J.M. O'Donnell ${ }^{4}$, R. Reifarth ${ }^{4}$, \\ R.S. Rundberg ${ }^{4}$, J.M. Schwantes ${ }^{4}$, J.L. Ullmann ${ }^{4}$, D.J. Vieira ${ }^{4}$, J.M. Wouters ${ }^{4}$, and P.A. Wilk ${ }^{1}$ \\ 1 Lawrence Livermore National Laboratory, Livermore, CA 94551, USA \\ 2 North Carolina State University, Raleigh, NC 27695, USA presently at Lawrence Livermore National Laboratory \\ 3 Charles University in Prague, 180000 Prague 9, Czech Republic \\ ${ }^{4}$ Los Alamos National Laboratory, Los Alamos, NM 87545, USA \\ 5 North Carolina State University, Raleigh, NC 27695, USA and Triangle Universities Nuclear Laboratory, Durham, NC 27708, USA
}

\begin{abstract}
We have made neutron capture cross-section measurements using the white neutron source at the Los Alamos Science Center, the DANCE detector array (Detector for Advanced Neutron Capture Experiments) and targets important for basic science and stockpile stewardship. In this paper, we review results from (n, $\gamma$ ) reactions on ${ }^{94,95} \mathrm{Mo}$, 152,154,157,160,nat $\mathrm{Gd},{ }^{151,153} \mathrm{Eu}$ and ${ }^{242 \mathrm{~m}} \mathrm{Am}$ for neutron energies from $<1 \mathrm{eV}$ up to $\sim 20 \mathrm{keV}$. We measured details of the $\gamma$-ray cascade following neutron capture, for comparison with results of statistical model simulations. We determined the neutron energy dependent $(\mathrm{n}, \gamma)$ cross section and gained information about statistical decay properties, including the nuclear level density and the photon strength function. Because of the high granularity of the detector array, it is possible to look at gamma cascades with a specified number of transitions (a specific multiplicity). We simulated $\gamma$-ray cascades using a combination of the DICEBOX/GEANT computer codes. In the case of the deformed nuclei, we found evidence of a scissors-mode resonance. For the Eu, we also determined the $(\mathrm{n}, \gamma)$ cross sections. For the ${ }^{94,95} \mathrm{Mo}$, we focused on the spin and parity assignments of the resonances and the determination of the photon strength functions for the compound nuclei ${ }^{95,96} \mathrm{Mo}$. Future plans include measurements on actinide targets; our immediate interest is in ${ }^{242 \mathrm{~m}} \mathrm{Am}$.
\end{abstract}

\section{Introduction}

Neutron-capture cross sections are important for basic nuclear science and for applied nuclear research, including stockpile science and technology. We now have a strong collaboration between the Lawrence Livermore National Laboratory, the Los Alamos National Laboratory, North Carolina State University and the Charles University in Prague. In this paper, we report on capture gamma measurements that are of particular interest to the Lawrence Livermore National Laboratory.

Our motivation is to improve the accuracy and provide new measurements or new energy ranges in capture cross-section data for stable and radioactive targets. We are especially interested in neutron energy-dependent capture cross sections. For each measured target, we performed a statistical study that included photon strength function, resonance parameters, level density and gamma-ray cascade multiplicity.

The work reported here includes ${ }^{94,95} \mathrm{Mo}(\mathrm{n}, \gamma)$ reactions and statistical studies, ${ }^{151,153} \mathrm{Eu}(\mathrm{n}, \gamma)$ cross-section measure-

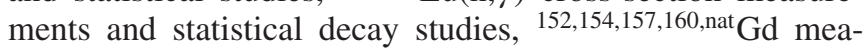
surements, and the first ${ }^{242 \mathrm{~m}} \mathrm{Am}(\mathrm{n}, \gamma)$ measurement.

\section{Experiment}

Measurements were performed at DANCE, the Detector for Advanced Neutron Capture Experiments. The detector array, made of 160 barium fluoride crystals arranged in a sphere, was designed to measure (n, $\gamma)$ cross sections with targets that were milligram sized or smaller, including radioactive targets [1]. The barium fluoride scintillators have a very fast response to light, and are therefore suitable for high count rate experiments. To reduce the background of scattered neutrons, a ${ }^{6} \mathrm{LiH}$ shell is placed inside the array. The detector array is located at the Lujan Center of the Los Alamos National Laboratory. The white neutron source provides thermal to $100 \mathrm{keV}$ neutrons, and incident neutron energy at the 20.25 meter flight path is determined by time-of-flight. The neutron source is a spallation neutron source with an intensity of $3 \times$ $10^{5} \mathrm{n} / \mathrm{s} / \mathrm{cm}^{2} /$ energy decade. The beam has a $20 \mathrm{~Hz}$ repetition rate, and the average beam current was $80 \mu \mathrm{A}$. The effective solid angle of the detector array is $3.52 \pi$, providing a gamma efficiency of about $90 \%$ and a cascade efficiency of about $98 \%$.

For the molybdenum experiments, the targets were selfsupporting molybdenum foils with thicknesses of $50 \mathrm{mg} / \mathrm{cm}^{2}$ and $25 \mathrm{mg} / \mathrm{cm}^{2}$ for ${ }^{94} \mathrm{Mo}$ and ${ }^{95} \mathrm{Mo}$, respectively [2].

The ${ }^{151,153} \mathrm{Eu}$ targets were enriched europium materials that were electroplated onto 0.5 mil beryllium foils. The ${ }^{151} \mathrm{Eu}$ was $96.83 \%$ enriched with a thickness of $0.836 \pm$ $0.040 \mathrm{mg} / \mathrm{cm}^{2}$, and the ${ }^{153} \mathrm{Eu}$ was $98.76 \%$ enriched with a thickness of $1.06 \pm 0.05 \mathrm{mg} / \mathrm{cm}^{2}$.

Several targets were used for the gadolinium measurements. The ${ }^{157,160} \mathrm{Gd}$ materials were very pure, while the ${ }^{152,154} \mathrm{Gd}$ targets were less pure. However the ${ }^{152,154} \mathrm{Gd}$ isotopes are significantly enriched above the natural abundance. We also made and measured a ${ }^{\text {nat }} \mathrm{Gd}$ target. A summary of the target materials is given in table 1 . In each case, the gadolinium material was electroplated onto 0.5 mil beryllium foils.

The ${ }^{242 \mathrm{~m}}$ Am material was made by irradiation of ${ }^{241} \mathrm{Am}$ in the Idaho National Laboratory's reactor. Samples were 
Table 1. Isotopic compositions, in percent, of the five Gd targets. The ${ }^{157,160} \mathrm{Gd}$ materials are very pure.

\begin{tabular}{llllll}
\hline Isotope & ${ }^{\text {nat }} \mathrm{Gd}$ & ${ }^{152} \mathrm{Gd}$ & ${ }^{154} \mathrm{Gd}$ & ${ }^{157} \mathrm{Gd}$ & ${ }^{160} \mathrm{Gd}$ \\
\hline${ }^{152} \mathrm{Gd}$ & 0.20 & 42.49 & 0.05 & $<0.01$ & $<0.01$ \\
${ }^{154} \mathrm{Gd}$ & 2.18 & 4.38 & 67.34 & $<0.01$ & 0.02 \\
${ }^{155} \mathrm{Gd}$ & 14.80 & 15.93 & 21.11 & 0.09 & 0.22 \\
${ }^{156} \mathrm{Gd}$ & 20.46 & 13.91 & 5.65 & 0.09 & 0.37 \\
${ }^{157} \mathrm{Gd}$ & 15.65 & 7.82 & 2.24 & 99.7 & 0.27 \\
${ }^{158} \mathrm{Gd}$ & 24.84 & 9.56 & 2.32 & 0.12 & 0.92 \\
${ }^{160} \mathrm{Gd}$ & 21.87 & 5.91 & 1.29 & $<0.01$ & 98.2 \\
\hline
\end{tabular}

recently purified and electroplated onto 0.5 mil beryllium foils. The total target mass for our initial measurement was about $47 \mu \mathrm{g}{ }^{242 \mathrm{~m}}$ Am material.

\section{Data analysis and experimental results}

\section{$3.1^{94,95} \operatorname{Mo}(\mathrm{n}, \gamma)$}

The ${ }^{94,95} \operatorname{Mo}(n, \gamma)$ reactions were studied for neutron energies from thermal to $16 \mathrm{keV}$. The primary motivation for these measurements was to test an enhancement observed in the low energy behavior of the photon strength function in ${ }^{93-98}$ Mo by the Oslo Cyclotron group [3].

Gamma-ray distribution and average gamma cascade multiplicities were measured following resonant neutron capture. These spectra were compared with simulations, which were performed with DICEBOX [4] and GEANT4. The DICEBOX output was the input for GEANT4. Models of the photon strength function with an Oslo-type enhancement below $1 \mathrm{MeV}$ were unable to reproduce experimental spectra. However, good agreement was found between DANCE spectra and simulations that postulate a low-lying resonance in the photon strength function; this is intermediate in strength between the KMF model and an Oslo-type photon strength function [2].

New spin assignments were made for resonances in the ${ }^{95,96}$ Mo excited nuclei. Assignments were made using the multiplicity of the $\gamma$-ray cascades and the $\gamma$-ray spectral shape. In the ${ }^{96} \mathrm{Mo}$ compound nucleus, 56 new resonances were observed. Seventeen resonances were given a new spin assignment, and 22 resonances were given a tentative spin assignment. For the ${ }^{95}$ Mo compound nucleus, 39 resonances were observed, and 21 resonances were given a parity assignment [2].

With the new resonance information, the resonance spacing can be improved. The new spacing, $\mathrm{D}_{0}$, is $82 \mathrm{eV}$, a change of more than $20 \%$ from $105 \pm 10 \mathrm{eV}$ in the Reference Input Parameter Library. The change in resonance spacing results in a cross-section change of as much as $40 \%$.

\section{$3.2^{151,153} \operatorname{Eu}(\mathrm{n}, \gamma)$}

The ${ }^{151,153} \operatorname{Eu}(\mathrm{n}, \gamma)$ reactions were studied because there was a large (30-40\%) difference between the existing data sets [5-7]. The historical data is shown in figure 1. All measurements shown in figure 1 were made with $\mathrm{C}_{6} \mathrm{D}_{6}$ detectors. The

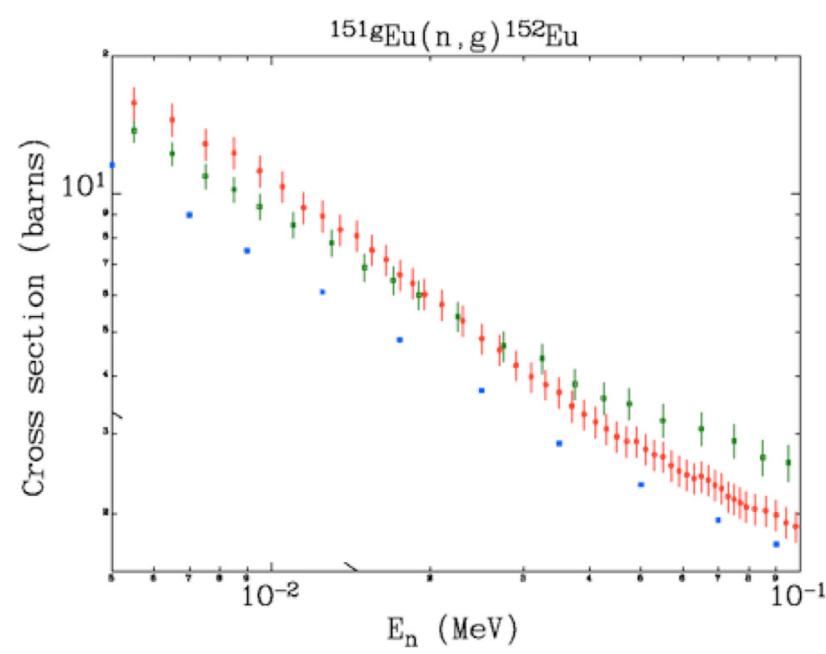

Fig. 1. Three measurements of ${ }^{151} \mathrm{Eu}(\mathrm{n}, \gamma)$ taken in 1977,1979 , and 1987. These measurements have a 30-40\% difference between them.

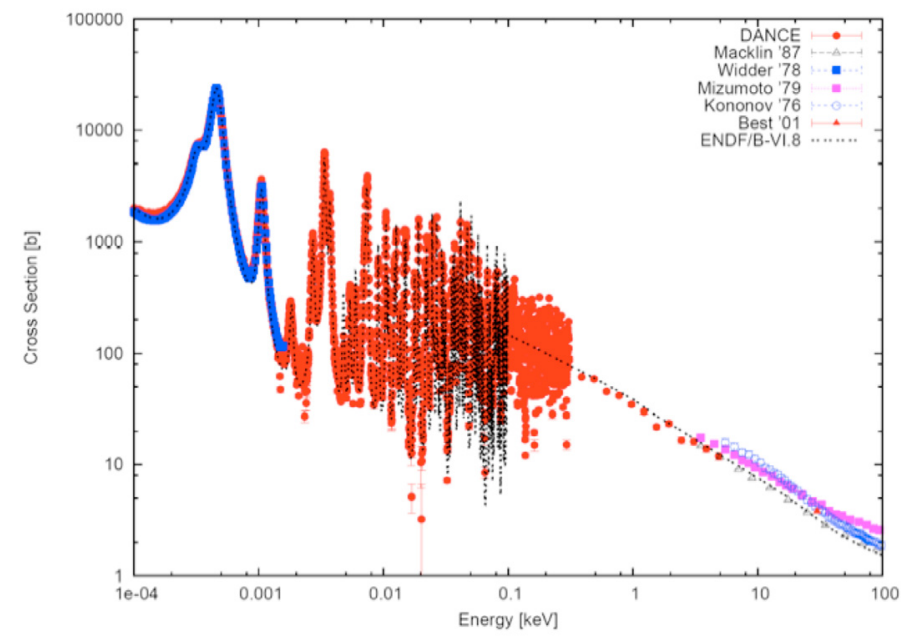

Fig. 2. Neutron capture cross section for ${ }^{151} \mathrm{Eu}$ from $0.2 \mathrm{eV}$ to $100 \mathrm{keV}$. The Macklin data (1987) is within the error range of the DANCE data.

evaluated cross-section set is currently an average of these measurements.

New ${ }^{151,153} \mathrm{Eu}$ measurements were made using the DANCE array. The instrumental $\gamma$-ray multiplicity of the detector array was utilized for the background subtraction, using the observation that events from the neutron capture of the ${ }^{151,153} \mathrm{Eu}$ targets are dominantly of multiplicity 3 and 4 , while events due to background are of low multiplicity [8]. Results suggest that we should reduce the evaluated cross section for incident neutrons above $1 \mathrm{keV}$. The entire neutron energy range is shown in figure 2, and the energy range from $0.1 \mathrm{keV}$ to $100 \mathrm{keV}$ is shown in figure 3 .

For ${ }^{151} \mathrm{Eu}$, the cross section was normalized to the wellknown doublet at $0.3-0.4 \mathrm{eV}$ measured by Widder et al. [9]. Similarly, the ${ }^{153} \mathrm{Eu}$ cross section (not shown) was normalized to the values determined by Widder. In figures 2 and 3, we also include data by Best et al. [10] which appears to agree with the Kononov data. 


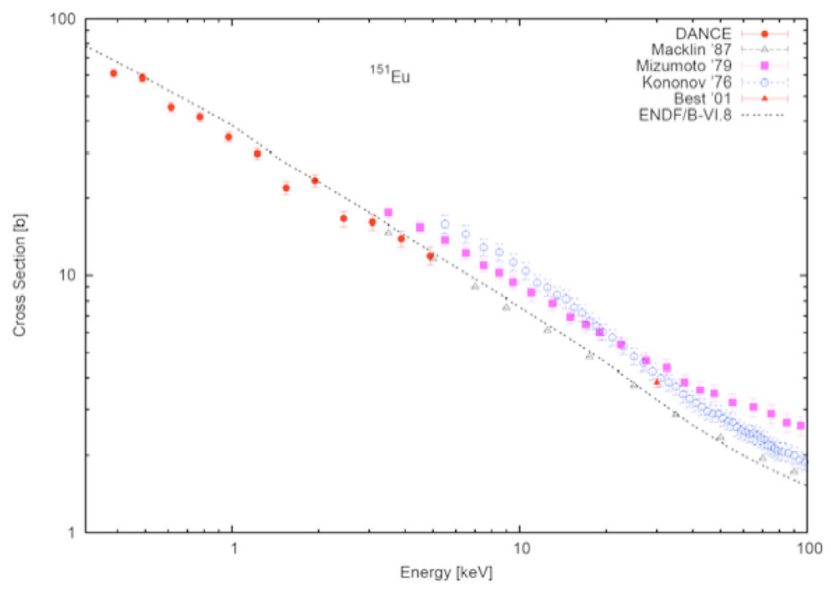

Fig. 3. Neutron capture cross section for ${ }^{151} \mathrm{Eu}$ from $0.3 \mathrm{keV}$ to $100 \mathrm{keV}$. The ENDF/B-VI calculation agrees with the new DANCE data and the Macklin data.

Various statistical properties of ${ }^{152,154} \mathrm{Eu}$ compound nuclei were investigated. In the case of the ${ }^{151,153}$ Eu targets, the multiplicity distributions were found to be independent of the incident neutron energy. The main finding of the statistical study was the photon strength function of ${ }^{152,154} \mathrm{Eu}$. Again, simulations were performed with DICEBOX [4] and GEANT4. The simulation performed with and without the scissorsmode resonance yielded noticeably different outcomes. To reproduce the experimental data, inclusion of the scissorsmode resonance was clearly necessary in simulations for both ${ }^{152} \mathrm{Eu}$ and ${ }^{154} \mathrm{Eu}$ compound nuclei [11]. An independent experiment to confirm this finding is now beginning at the Charles University in Prague and the Nuclear Physics Institute (in Rez).

\section{$3.3{ }^{152,154,157,160} \mathrm{Gd}(\mathrm{n}, \gamma)$ and ${ }^{\mathrm{nat}} \mathrm{Gd}(\mathrm{n}, \gamma)$}

The gadolinium data is similar to the europium data in that there are several historical measurements, which do not agree with each other. In particular there is some difference within the ${ }^{154} \mathrm{Gd}(\mathrm{n}, \gamma)$ data. Existing ${ }^{152} \mathrm{Gd}$ has better self-agreement, but this data still does not extend to energies below $3 \mathrm{keV}$.
We have completed ${ }^{152,154,157} \mathrm{Gd}$ as well as the ${ }^{\text {nat }} \mathrm{Gd}$ measurements, and data analysis is currently underway. The next measurement will be on ${ }^{160} \mathrm{Gd}$, which is planned for July of 2007.

Our gadolinium target materials have various levels of purity, as shown in table 1 . In the case of ${ }^{152,154} \mathrm{Gd}$, we will have background subtractions from other isotopes of gadolinium to consider, and these subtractions will become difficult above the resonance region. It is important to note that the ${ }^{152,154} \mathrm{Gd}$ materials are highly enriched compared to their natural abundances; resonance information we gain may be significant.

\section{$3.4{ }^{242 m} \mathrm{Am}(\mathrm{n}, \gamma)$}

The next measurement we expect to do is ${ }^{242 \mathrm{~m}} \mathrm{Am}(\mathrm{n}, \gamma)$. There is currently no capture cross-section data for this isotope, which is an odd-odd actinide. The anticipated cross section is $>1$ barn below the incident neutron energy of $1 \mathrm{keV}$.

For all the actinide targets, neutron bombardment results in both fission and capture reactions. Los Alamos National Laboratory, in a collaboration with Lawrence Livermore National Laboratory, has developed a fission-tagging detector, [12] which reduces the "background" from the fission and $\alpha$-decay events during $(\mathrm{n}, \gamma)$ measurements. For the capture reactions, most $\gamma$ rays associated with the $(\mathrm{n}, \mathrm{f})$ reactions can be removed using the fission tagging detector. Alternatively, the fission tagging detector allows simultaneous measurement of $(\mathrm{n}, \mathrm{f})$ and $(\mathrm{n}, \gamma)$ cross sections.

We made a preliminary measurement on a $47 \mu \mathrm{g}$ target in September of 2006. With this thin target, we were able to make a good ${ }^{242 \mathrm{~m}} \mathrm{Am}(\mathrm{n}, \mathrm{f})$ measurement. After subtraction of the $\gamma$ rays associated with fission, we found that the $(\mathrm{n}, \gamma)$ data was barely above the background. The fission crosssection results are shown in figure 4 . Above $7 \mathrm{eV}$, the DANCE experimental data is in excellent agreement with the previous neutron induced fission cross-section measurements done by J. Browne et al. [13]. Discrepancies between the DANCE data and the Browne data are currently under investigation.

During 2007, we plan to make a new ${ }^{242 \mathrm{~m}}$ Am target by electroplating material onto titanium foils, and we expect that

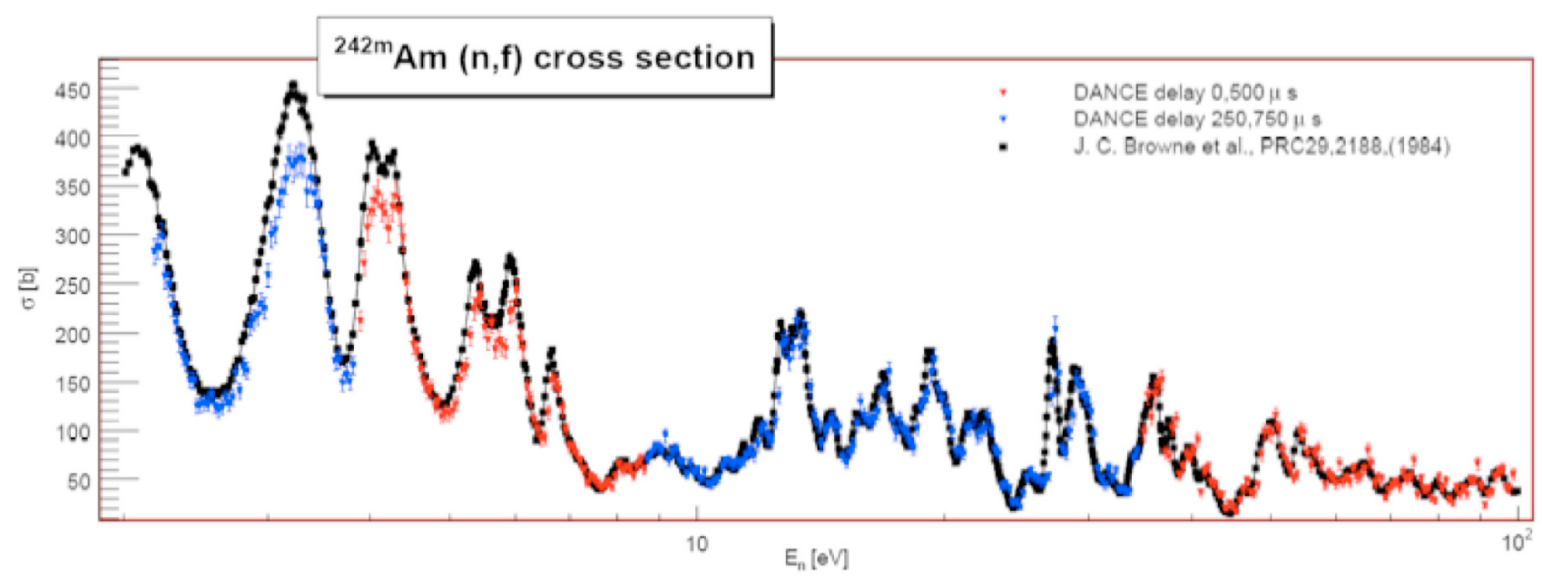

Fig. 4. The ${ }^{242 m} \mathrm{Am}(\mathrm{n}, \mathrm{f})$ cross section measured at the DANCE detector is shown with the data from J. Browne et al. 
the total target material will be $400 \mu \mathrm{g}$ of ${ }^{242 \mathrm{~m}} \mathrm{Am}$. Our (n, $\gamma$ ) measurement is planned for November 2007.

\section{Conclusions}

In summary, DANCE is an excellent facility for determining $(\mathrm{n}, \gamma)$ cross sections and for studying statistical decay of excited nuclei. The molybdenum experiment was a good test case for improving the spin assignments, and we were able to contribute to the discussion regarding the molybdenum strength function. We were able to determine the ${ }^{151,153} \mathrm{Eu}$ neutron capture cross sections, and we used our data for statistical decay studies. Neutron capture data has been collected for several gadolinium isotopes. The Gd data will also be used for statistical decay studies and improved cross sections. Using the fission tagging detector, we made a new measurement of ${ }^{242 \mathrm{~m}} \mathrm{Am}(\mathrm{n}, \mathrm{f})$. The ${ }^{242 \mathrm{~m}} \mathrm{Am}(\mathrm{n}, \gamma)$ measurement will be the first of an odd-odd actinide.

This work was performed under the auspices of the US Department of Energy by the University of California, Lawrence Livermore National Laboratory under contract No. W-7405-ENG-48, and by Los Alamos National Security, LLC, Los Alamos National Laboratory under contract No. DE-AC52-06NA25396. This work was supported in part by the US Department of Energy Grants No. DE-FG0303NA00076 and No. DE-FG02-97-ER41042.

\section{References}

1. M. Heil, R. Reifarth, M.M. Fowler, R.C. Haight, F. Kappeler, R.S. Rundberg, E.H. Seabury, J.L. Ullmann, J.B. Wilhelmy, K. Wisshak, NIM A 459, 229 (2001).

2. S.A. Sheets, Ph.D. thesis, North Carolina State University, 2006.
3. M. Guttormsen, R. Chankova, U. Agvaanluvsan, E. Algin, L.A. Bernstein, F. Ingebretsen, T. Lonnroth, S. Messelt, G.E. Mitchell, J. Rekstad, A. Schiller, S. Siem, A.C. Sunde, A. Voinov, S. Odegard, Phys. Rev. C 71, 044307 (2005).

4. F. Becvar, NIM A 417, 434 (1998).

5. R.L. Macklin, P.G. Young, Nucl. Sci. Eng. 95, 189 (1987).

6. M. Mizumoto, A. Asami, Y. Nakajima, T. Fuketa, H. Takekoshi, J. Nucl. Sci. Technol. (Tokyo) 16, 711 (1979).

7. V.N. Kononov, B.D Yurlov, Proceedings of the 4th All-Union Conf. Nucl. Phys., Kiev, April 18-22 (1977).

8. U. Agvaanluvsan, J.A. Becker, R.A. Macri, W.E. Parker, P.A. Wilk, C.Y. Wu, T.A. Bredeweg, E. Esch, R.C. Haight, J.M. O’Donnell, R. Reifarth, R.S. Rundberg, J.M. Schwantes, J.L. Ullmann, D.J. Vieira, J.B. Wilhelmy, J.M. Wouters, G.E. Mitchell, S.A. Sheets, M. Krticka, F. Becvar (in preparation).

9. F. Widder, Eidg. Inst. Reaktorforsch. Wuerenlingen Reports No. 217 (1975).

10. J. Best, H. Stoll, C. Arlandini, S. Jaag, F. Kappeler, K. Wisshak, A. Mengoni, G. Reffo, T. Rauscher, Phys. Rev. C 64, 015801 (2001).

11. U. Agvaanluvsan, A. Alpizar-Vicente, J.A. Becker, F. Becvar, T.A. Bredeweg, R. Clement, E. Esch, R. Hatarik, R.C. Haight, M. Krticka, R.A. Macri, G.E. Mitchell, J.M. O'Donnell, W.E. Parker, R. Reifarth, R.S. Rundberg, J.M. Schwantes, S.A. Sheets, D.J. Vieira, J.B. Wilhelmy, J.L. Ullmann, P. Wilk, $12^{\text {th }}$ Conference on Capture Gamma-Ray Spectroscopy, Vol. 12, Sept. 2005.

12. T.A. Bredeweg, J.A. Becker, E. Bond, M.M. Fowler, R.C. Haight, M. Jandel, P. Koehler, R. Macri, J. O'Donnell, W.E. Parker, R. Reifarth, R.S. Rundberg, J.L. Ullmann, D.J. Vieira, J. Wilhelmy (these proceedings).

13. J.C. Browne, R.M. White, R.E. Howe, J.H. Landrum, R.J. Dougan, R.J. Dupzyk, Phys. Rev. C 29, 2188 (1984). 